\title{
Temperature-Driven Oxygenation Rate Control by Polymeric Photosensitizer
}

\author{
Hisao Koizumi, ${ }^{\dagger}$ Yasuhiro Shiraishi, ${ }^{* \dagger}$ Sachiko Tojo, ${ }^{*}$ Mamoru Fujitsuka, ${ }^{\star}$ \\ Tetsuro Majima, ${ }^{*}$ and Takayuki Hirai ${ }^{\dagger}$ \\ Research Center for Solar Energy Chemistry, and Division of Chemical Engineering, Graduate School of \\ Engineering Science, Osaka University, Toyonaka, Osaka 560-8531, and The Institute of Scientific and \\ Industrial Research (SANKEN), Osaka University, Mihogaoka 8-1, Ibaraki, Osaka 565-0047, Japan
}

\section{Supporting Information}

\section{Materials}

Poly(NIPAM-co-BP): NIPAM (0.60 g, $5.3 \mathrm{mmol})$, 4-allyloxyBP ${ }^{[\mathrm{S} 1]}(0.13 \mathrm{~g}, 0.53 \mathrm{mmol})$, and AIBN $(0.017 \mathrm{~g}, 0.11 \mathrm{mmol})$ were dissolved in toluene $(5 \mathrm{~mL})$. The solution was degassed by twice freeze-pump-thaw cycles. The solution was heated to $60{ }^{\circ} \mathrm{C}$ under dry $\mathrm{N}_{2}$ for $18 \mathrm{~h}$, and cooled to room temperature. The resulting mixture was added slowly to diethyl ether $(100 \mathrm{~mL})$ with magnetic stirring. The precipitate formed was collected by centrifugation and purified by re-precipitation with $\mathrm{MeOH}(1 \mathrm{~mL})$ and diethyl ether $(100 \mathrm{~mL})$, affording poly(NIPAM-co-BP) as a fluffy white solid (0.52 g, yield 71\%): $\delta_{\mathrm{H}}(\mathrm{DMSO}, 270 \mathrm{MHz}): 1.07$ (s, br, $\left.-\mathrm{C}\left(\mathrm{CH}_{3}\right)_{2}\right), 1.2-2.2(\mathrm{~m}$, ${ }_{-} \mathrm{CHCH}_{2}-$ ), 3.88 (s, br, $-\mathrm{CH}-$ ), 6.72 (br, $\left.-\mathrm{N} H-\right), 7.65$ (br, Ar- $H$ ). The quantity of the BP unit on the polymer was determined by comparison of absorbance $\left(A_{290}\right)$ with 4-methoxyBP $(\varepsilon=7.98$ $\mathrm{mM}^{-1} \mathrm{~cm}^{-1}$ ) dissolved in $\mathrm{MeOH}$ at $298 \mathrm{~K}$ (Figure S7). The ratio of the NIPAM/BP units on poly $\left(\mathrm{NIPAM}_{x}-c o-\mathrm{BP}_{y}\right)$ was therefore estimated to be $x: y=0.94: 0.06 . M_{\mathrm{n}}(\mathrm{GPC})=71300$.

PolyNIPAM (BP-free): This was synthesized according to literature procedure, ${ }^{[\mathrm{S} 2]}$ as follows: NIPAM (0.50 g, $4.4 \mathrm{mmol})$ and AIBN (0.014 g, $0.088 \mathrm{mmol})$ were dissolved in toluene $(5 \mathrm{~mL})$. The solution was degassed by twice freeze-pump-thaw cycles. The solution was heated to $60{ }^{\circ} \mathrm{C}$ under dry $\mathrm{N}_{2}$ for $15 \mathrm{~h}$, and cooled to room temperature. The resulting mixture was added slowly to diethyl ether $(100 \mathrm{~mL})$ with magnetic stirring. The precipitate formed was collected by centrifugation and purified by re-precipitation with $\mathrm{MeOH}(1 \mathrm{~mL})$ and diethyl ether $(100 \mathrm{~mL})$, affording polyNIPAM as a fluffy white solid (0.35 g, yield 71\%): $\delta_{\mathrm{H}}(\mathrm{DMSO}, 270 \mathrm{MHz}): 1.07$ (s, br, $\left.6 \mathrm{H},-\mathrm{C}\left(\mathrm{CH}_{3}\right)_{2}\right), 1.2-2.2\left(\mathrm{~m}, 3 \mathrm{H},-\mathrm{C} H \mathrm{CH}_{2}-\right), 3.84$ (s, br, $\left.1 \mathrm{H},-\mathrm{CH}-\right), 6.73$ (br, $\left.1 \mathrm{H},-\mathrm{NH}-\right)$. $M_{\mathrm{n}}$ $(\mathrm{GPC})=71600$. 


\section{Methods}

Photooxygenation. Each sensitizer was dissolved in a buffered aqueous solution $(5 \mathrm{~mL} ; \mathrm{pH}$ 10; consisting of $0.025 \mathrm{M} \mathrm{NaHCO}_{3}$ and $0.011 \mathrm{M} \mathrm{NaOH}$ ) containing 1 within a Pyrex glass tube (capacity: $20 \mathrm{~mL}$ ). Each tube was sealed using a rubber septum cap. $\mathrm{O}_{2}$ was bubbled through the solution for $5 \mathrm{~min}$ at $5{ }^{\circ} \mathrm{C}$ to avoid solvent evaporation. The sample was photoirradiated by a high-pressure Hg lamp (100 W; Eikohsha Co. Ltd., Osaka, Japan), filtered through an aqueous $\mathrm{CuSO}_{4}(10 \mathrm{wt} \%)$ solution to give light wavelengths of $\lambda>320 \mathrm{~nm}$, with magnetic stirring in a temperature range of $5-35{ }^{\circ} \mathrm{C}$ [light intensity at $320-400 \mathrm{~nm}$ (through the filter), $905 \mathrm{~mW} / \mathrm{m}^{2}$ ]. After photoirradiation, the solution was analyzed by gas chromatography (Shimadzu GC-14B, equipped with FID).

ESR measurement. The spectra were recorded at the X-band using a Bruker EMX-10/12 spectrometer ${ }^{[\mathrm{S3}, \mathrm{S} 4]}$ (scan conditions: microwave frequency, $9.7 \mathrm{GHz}$; microwave power, $10 \mathrm{~mW}$; modulation amplitude, $1.0 \mathrm{G}$; modulation frequency, $100 \mathrm{kHz}$; time constant, $0.66 \mathrm{~s}$; scan time, $335 \mathrm{~s}$; receiver gain, $4.0 \times 10^{5}$; center field setting at $3455 \mathrm{G}$ ), where microwave power saturation of the signals does not occur. 1,1'-Diphenyl-2-picrylhydrazyl (DPPH) was used for the magnetic field calibration. TEMP $(250 \mu \mathrm{mol})$ and sensitizer were dissolved in a buffered aqueous solution ( $5 \mathrm{~mL} ; \mathrm{pH} 10$ ) within a Pyrex glass tube, and $\mathrm{O}_{2}$ was bubbled through the solution for $5 \mathrm{~min}$ at 5 ${ }^{\circ} \mathrm{C}$. The tube was photoirradiated for $10 \mathrm{~min}$ by a high-pressure $\mathrm{Hg}$ lamp in a similar manner to the photooxygenation experiment. After photoirradiation, the solution was introduced to a conventional quartz ESR tube, and measurement was then started at room temperature. ${ }^{[\mathrm{S} 5]}$

Laser Photolysis Measurement. Transient absorption spectra were measured using a photodiode array detector (Hamamatsu Photonics, S3904-1024F) with a gated image intensifier (Hamamatsu Photonics, C2925-01). The monitor light was obtained from a pulsed 450-W Xe arc lamp (Ushio, UXL-451-0), which was operated by a large current pulsed-power supply synchronized with the laser. The monitor light was passed through an iris with a diameter of 0.2 $\mathrm{cm}$ and sent into the sample solution perpendicular to the laser pulse. The monitor light passing through the sample was focused on the entrance slit of a monochromator (Unisoku, MD200) and detected with a photomultiplier tube (Hamamatsu Photonics, R2949). A $\mathrm{Nd}^{3+}:$ YAG laser (Brilliant, Quantel) was used for $355 \mathrm{~nm}$ excitation. Each single pulse lasted $5 \mathrm{~ns}$ with energy of 8 $\mathrm{mJ}$ per pulse. Laser photolysis measurement of an aqueous solution $(\mathrm{pH} 10 ; 3 \mathrm{~mL})$ containing poly(NIPAM-co-BP) $\left(2.16 \mathrm{mg}\right.$ containing $1.08 \mu \mathrm{mol} \mathrm{BP}$ unit; $\left.\varepsilon_{355}=436 \mathrm{M}^{-1} \mathrm{~cm}^{-1}\right)$ does not show 
any transient absorption at entire wavelength range $(400-850 \mathrm{~nm})$. This is because $\mathrm{H}_{2} \mathrm{O}$ molecules strongly quench the transient species. We therefore selected $\mathrm{CHCl}_{3}$ as the solvent for measurements.

Other Analysis. Absorption spectra were recorded on an UV-visible photodiode-array spectrophotometer (Shimadzu; Multispec-1500) with a $10 \mathrm{~mm}$ path length quartz cell. ${ }^{1} \mathrm{H}$ NMR spectra were obtained by JEOL JNM-AL270 with TMS as standard. Fluorescence and phosphorescence spectra $(77$ K) were measured on a Hitachi F-4500 fluorescence spectrophotometer, where both excitation and emission slit width of $2.5 \mathrm{~nm}$ was employed. The measurement was done within a $6 \mathrm{~mm}$ cylindrical quartz tube. Dynamic light scattering (DLS) measurements were carried out with a laser scattering spectrometer (LB-500, HORIBA), where the light source was a $5 \mathrm{~mW}$ semiconductor laser $(\lambda=650 \mathrm{~nm})$ and the scattering angle was $90^{\circ}$. Molecular weight of the polymers were determined by a gel permeation chromatography (GPC) using a JASCO HPLC system equipped with a PU-980 pump (JASCO) and a refractive index detector RI-930 (JASCO), with KF-806L column (Shodex). The oven temperature was $40{ }^{\circ} \mathrm{C}$, and DMF containing $\operatorname{LiBr}(0.01 \mathrm{M})$ was used as the carrier solvent (flow rate: $0.6 \mathrm{~mL} / \mathrm{min}){ }^{[\mathrm{S} 6]}$ 
Table S1. Lifetime $\left(\tau_{1 \mathrm{O} 2}\right)$ and diffusion coefficient $\left(D_{1 \mathrm{O} 2}\right)$ of singlet oxygen in various solvents (literature values).

\begin{tabular}{|c|c|c|}
\hline solvents & $\begin{array}{l}\tau_{102} \\
(\mu \mathrm{s})\end{array}$ & $\begin{array}{c}D_{1 \mathrm{O} 2} \\
\left(10^{-5} \mathrm{~cm}^{2} \mathrm{~s}^{-1}\right)\end{array}$ \\
\hline$n$-hexane ${ }^{a}$ & $31.4^{[\mathrm{S} 7]}$ & \\
\hline$n$-heptane ${ }^{a}$ & $29.5^{[\mathrm{S} 7]}$ & \\
\hline$n$-nonane ${ }^{a}$ & $23.7^{[\mathrm{S} 7]}$ & \\
\hline cyclohexane & $23^{[\mathrm{S} 8], b}$ & $5.31^{[\mathrm{S} 9], c}$ \\
\hline benzene & $31.2^{[\mathrm{S} 7], a}$ & $2.89^{[\mathrm{S} 9], c}$ \\
\hline methanol $^{a}$ & $10.4^{[\mathrm{S} 7]}$ & \\
\hline ethanol & $15.3^{[\mathrm{S} 7], a}$ & $2.64^{[\mathrm{S} 9], c}$ \\
\hline 1-propanol ${ }^{a}$ & $16.3^{[\mathrm{S} 7]}$ & \\
\hline 1-butanol ${ }^{a}$ & $17.5^{[\mathrm{S} 7]}$ & \\
\hline $\mathrm{H}_{2} \mathrm{O}\left(10^{\circ} \mathrm{C}\right)$ & $4.2^{[\mathrm{S} 7]}$ & $1.7^{[\mathrm{S} 10]}$ \\
\hline $\mathrm{H}_{2} \mathrm{O}\left(20^{\circ} \mathrm{C}\right)$ & & $2.3^{[\mathrm{S} 10]}$ \\
\hline $\mathrm{H}_{2} \mathrm{O}\left(30^{\circ} \mathrm{C}\right)$ & & $3.49^{[\mathrm{S} 8]}, 2.8^{[\mathrm{S} 10]}$ \\
\hline $\mathrm{H}_{2} \mathrm{O}\left(40{ }^{\circ} \mathrm{C}\right)$ & & $3.8^{[\mathrm{S} 10]}$ \\
\hline $\mathrm{H}_{2} \mathrm{O}\left(50^{\circ} \mathrm{C}\right)$ & & $4.2^{[\mathrm{S} 10]}$ \\
\hline $\mathrm{H}_{2} \mathrm{O}\left(60{ }^{\circ} \mathrm{C}\right)$ & & $5.7^{[\mathrm{S} 10]}$ \\
\hline $\mathrm{D}_{2} \mathrm{O}$ & $65^{[\mathrm{S} 11], b}, 55^{[\mathrm{S} 7], a}$ & $3^{[\mathrm{S} 11], b}$ \\
\hline \multicolumn{3}{|l|}{${ }^{a} 22^{\circ} \mathrm{C}$} \\
\hline \multicolumn{3}{|l|}{${ }^{b}$ room temp. } \\
\hline $29.6^{\circ} \mathrm{C}$ & & \\
\hline
\end{tabular}


Table S2. Lifetime $\left(\tau_{102}\right)$ and diffusion coefficient $\left(D_{102}\right)$ of singlet oxygen in polymer matrix (literature values).

\begin{tabular}{|c|c|c|}
\hline polymer & $\begin{array}{l}\tau_{1 \mathrm{O} 2} \\
(\mu \mathrm{s})\end{array}$ & $\begin{array}{c}D_{1 \mathrm{O} 2} \\
\left(10^{-7} \mathrm{~cm}^{2} \mathrm{~s}^{-1}\right)\end{array}$ \\
\hline \multirow[t]{4}{*}{ polystyrene glass } & $21^{[\mathrm{S} 12]}$ & \\
\hline & $20^{[\mathrm{S} 13]}$ & \\
\hline & & $2^{[\mathrm{S} 14]}$ \\
\hline & & $0.1-3.3^{[\mathrm{S} 17]}$ \\
\hline \multirow[t]{2}{*}{ Polystyrene } & $17-21^{[\mathrm{S} 13]}$ & \\
\hline & & $2.1-4.3^{[\mathrm{S} 10]}$ \\
\hline polystyrene film ${ }^{[\mathrm{S} 14]}$ & & $1.4-3.4$ \\
\hline poly(methylmethacrylate) glass ${ }^{[\mathrm{S} 13]}$ & $20-25$ & \\
\hline $\operatorname{poly}(p$-phenylene $)$ in benzene ${ }^{[\mathrm{S} 15]}$ & 29 & \\
\hline polyvinyl alcohol ${ }^{[\mathrm{S} 16]}$ & 10.3 & \\
\hline polyvinyl chloride ${ }^{[\mathrm{S} 16]}$ & 28.1 & \\
\hline polyvinyl acetate ${ }^{[\mathrm{S} 16]}$ & 31.4 & \\
\hline methyl cellulose ${ }^{[\mathrm{S} 16]}$ & 20.6 & \\
\hline polycarbonate films ${ }^{[\mathrm{S} 14]}$ & & $0.31-1.1$ \\
\hline poly(ethylene-co-norbornene) ${ }^{[\mathrm{S} 18]}$ & & $0.22-0.58$ \\
\hline
\end{tabular}

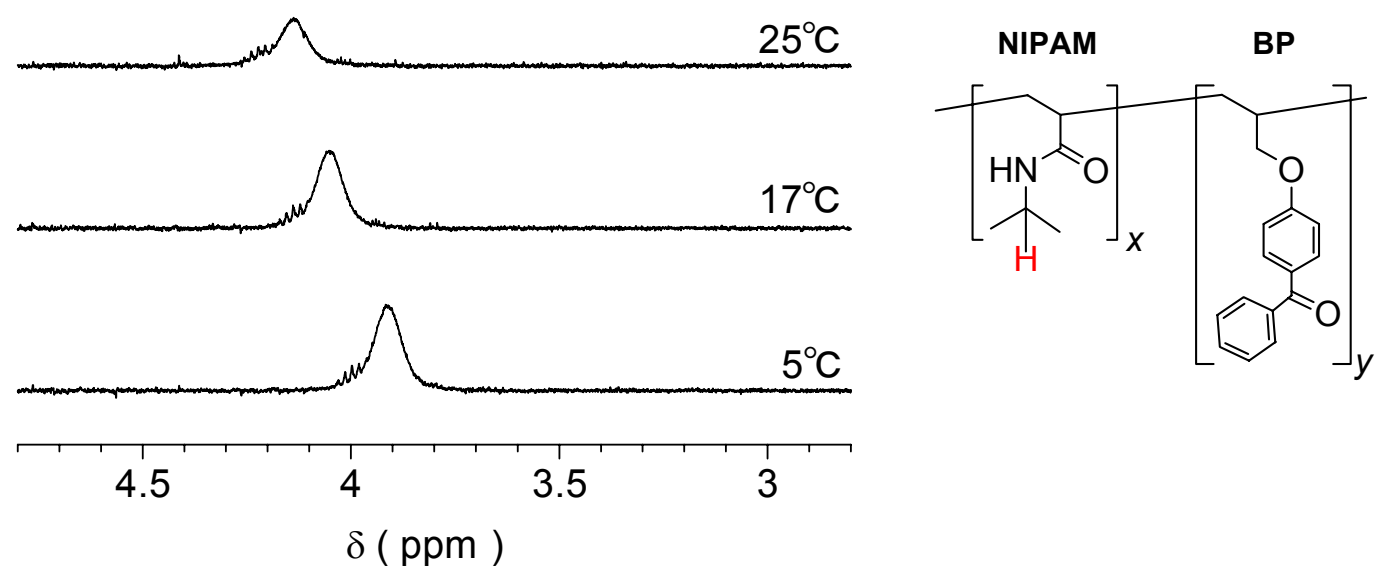

Figure S1. Change in ${ }^{1} \mathrm{H}$ NMR spectra of poly(NIPAM-co-BP) dissolved in $\mathrm{D}_{2} \mathrm{O}(\mathrm{pH}$ 10) with temperature. 


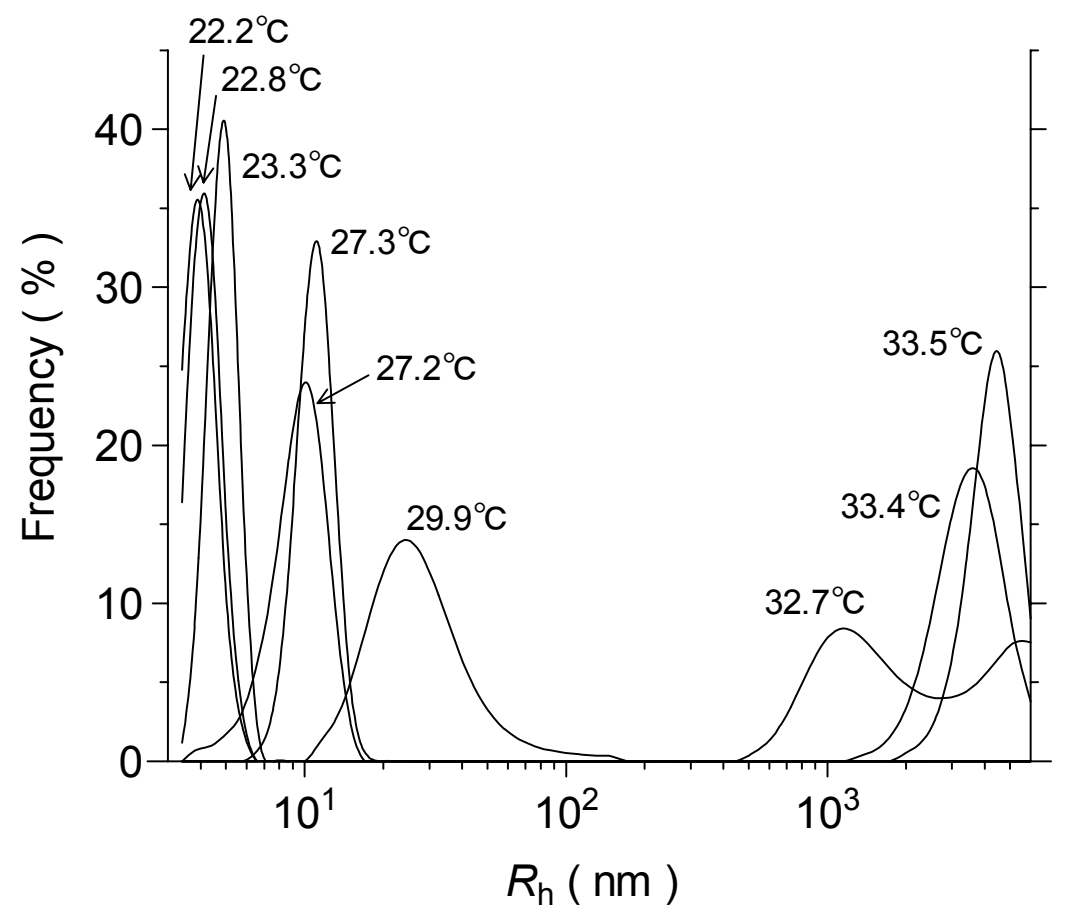

Figure S2. Change in distribution of hydrodynamic radius $\left(R_{\mathrm{h}}\right)$ of poly(NIPAM-co-BP) in aqueous solution ( $\mathrm{pH} 10)$ with temperature.

A

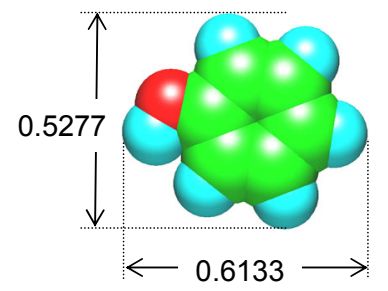

C

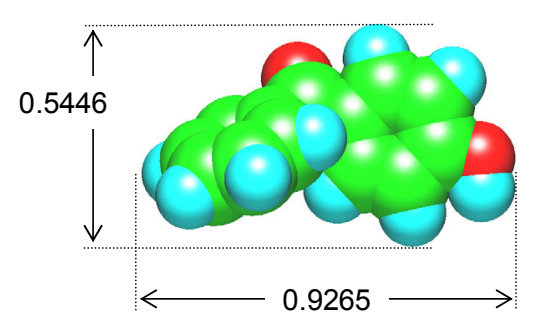

B

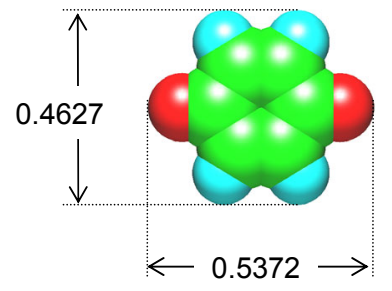

D

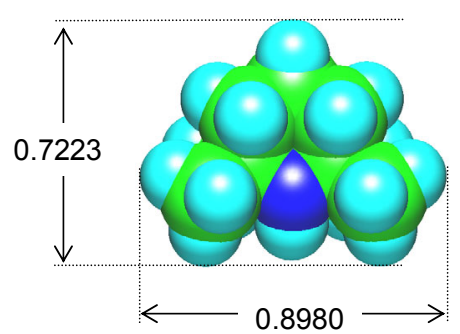

Figure S3. Maximum length of molecules and its perpendicular length, as determined by semiempirical MO calculation (PM3) on the WinMOPAC ver.3.0 software (unit: $\mathrm{nm}$ ). ${ }^{[\mathrm{S} 3]}$ Molecules are: (A) 1, (B) 2, (C) HBP, and (D) TEMP. 


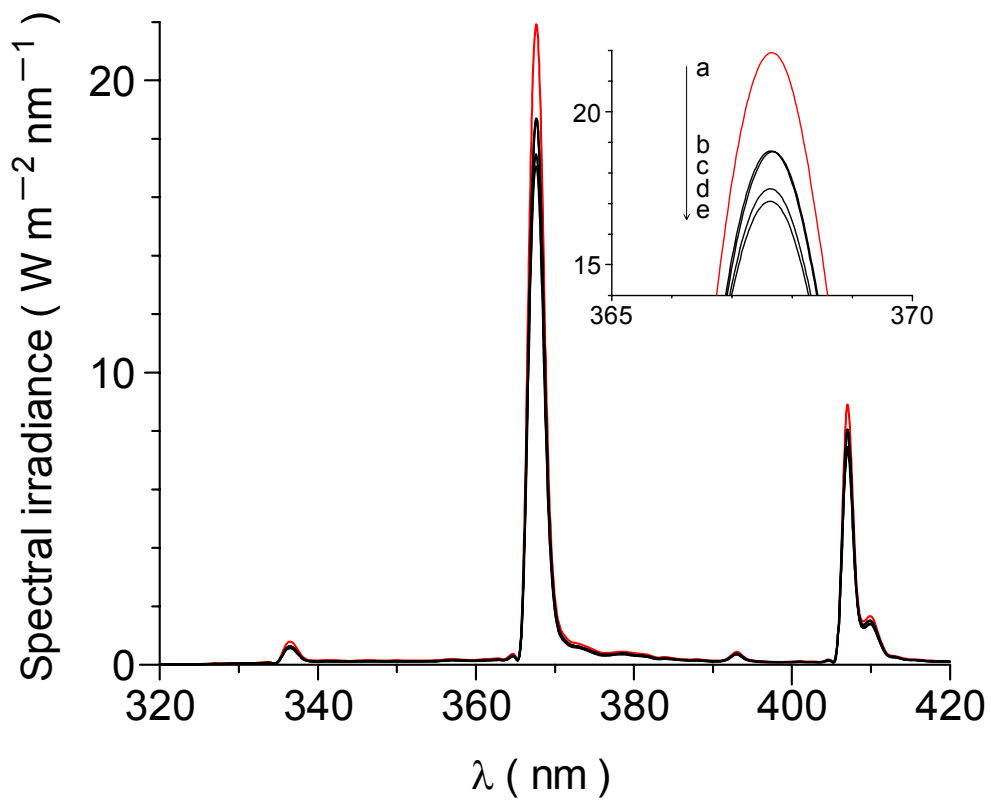

Figure S4. Change in spectral irradiance measured (a) behind the $\mathrm{CuSO}_{4}$ filter and behind the aqueous solution ( $\mathrm{pH} \mathrm{10)}$ containing $1(10 \mu \mathrm{mol})$ and poly(NIPAM-co-BP) $(0.2 \mathrm{mg})$ at (b) $5{ }^{\circ} \mathrm{C}$, (c) $17{ }^{\circ} \mathrm{C}$, (d) $25{ }^{\circ} \mathrm{C}$, and (e) $35{ }^{\circ} \mathrm{C}$. Transmittance of the solutions is: (b) $85.3 \%$, (c) $85.1 \%$, (d) $83.1 \%$, and (e) $82.2 \%$, based on the transmittance of (a) as $100 \%$.

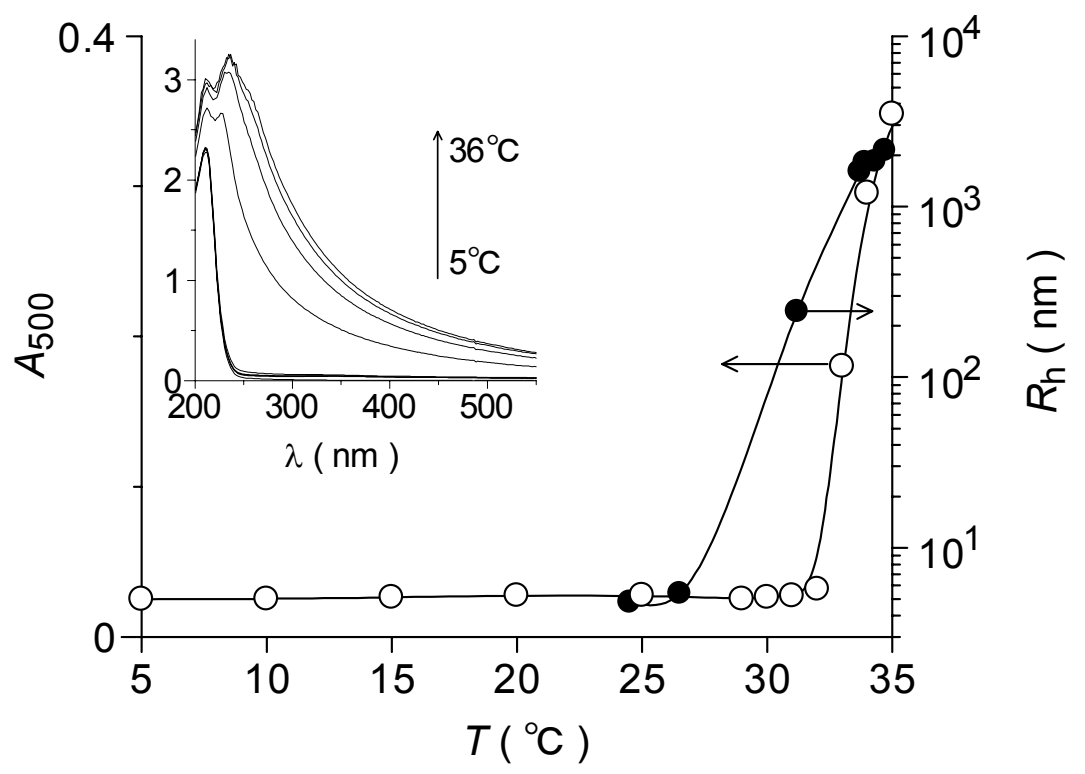

Figure S5. Change in turbidity $\left(A_{500}\right)$ and hydrodynamic radius $\left(R_{\mathrm{h}}\right)$ of polyNIPAM in aqueous solution ( $\mathrm{pH} \mathrm{10)}$ with temperature. (Inset) Change in absorption spectra with temperature. Detailed $R_{\mathrm{h}}$ data: see Figure $\mathrm{S} 6$. 


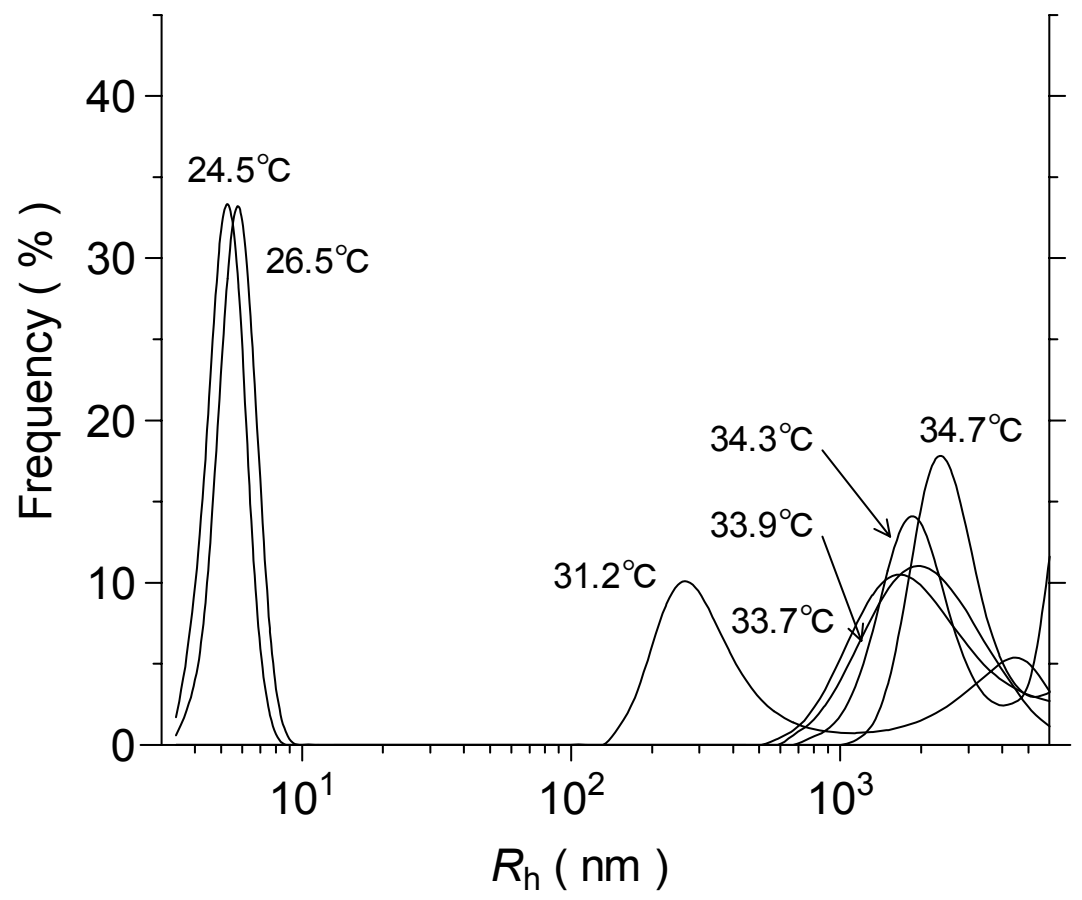

Figure S6. Change in distribution of hydrodynamic radius $\left(R_{\mathrm{h}}\right)$ of polyNIPAM in aqueous solution ( $\mathrm{pH} 10)$ with temperature.

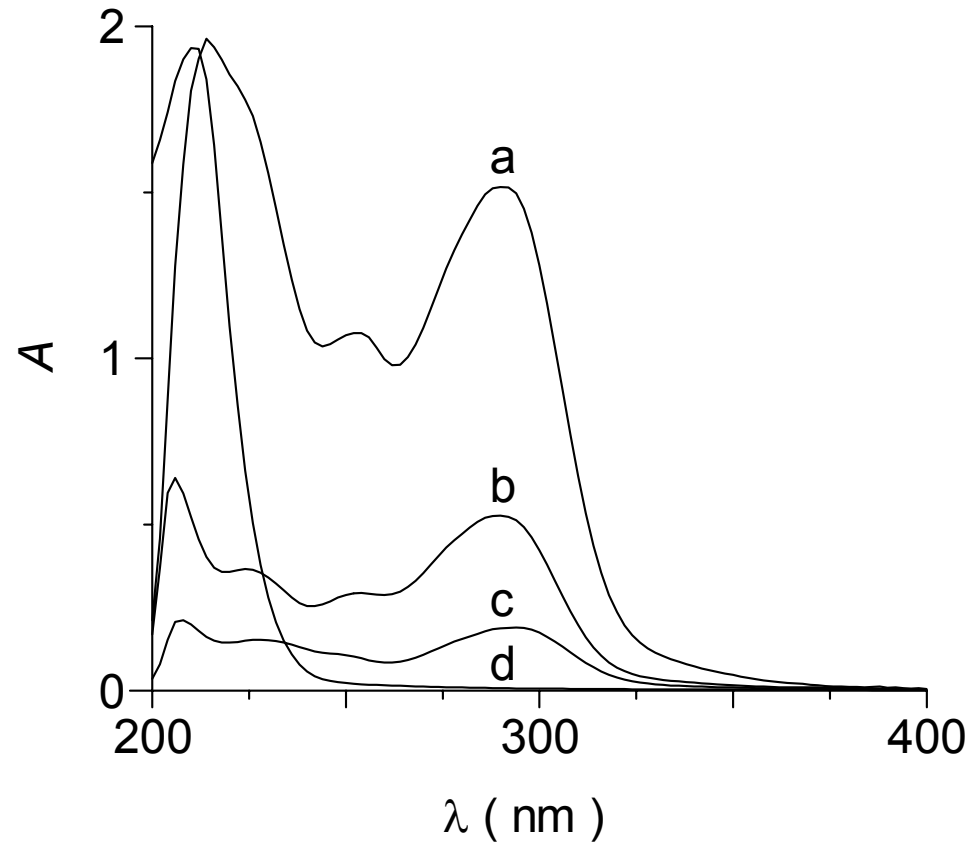

Figure S7. Absorption spectra of (a) poly(NIPAM-co-BP) $(0.38 \mathrm{~g} / \mathrm{L})$, (b) 4-methoxyBP $(6.6 \times$ $\left.10^{-5} \mathrm{M}\right),(\mathrm{c}) \mathrm{HBP}\left(2 \times 10^{-5} \mathrm{M}\right)$, and $(\mathrm{d})$ polyNIPAM $(0.4 \mathrm{~g} / \mathrm{L})$ in $\mathrm{MeOH}$ at $298 \mathrm{~K}$. 

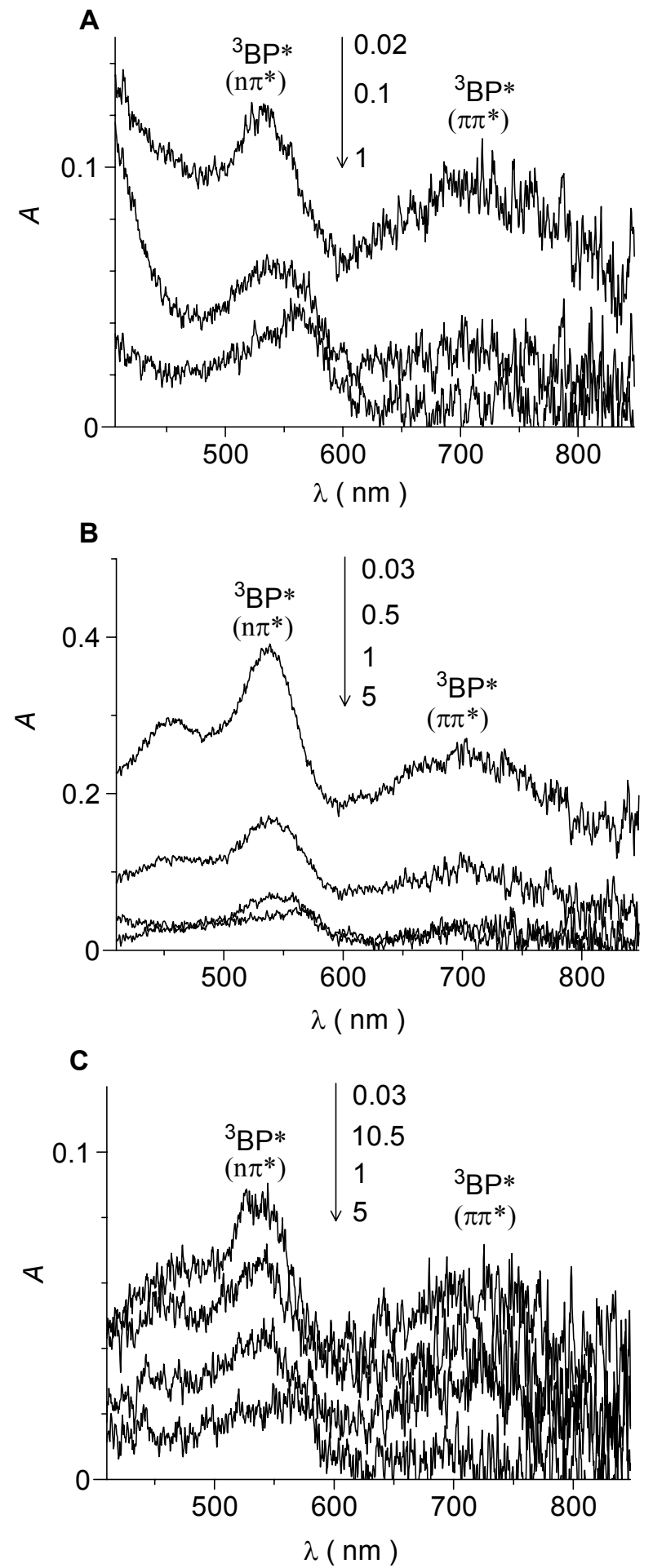

Figure S8. Transient absorption spectra ( $355 \mathrm{~nm}$ laser excitation; $298 \mathrm{~K}$; in $\mathrm{CHCl}_{3}(3 \mathrm{~mL})$ ) measured at different time intervals after photolysis (unit: $\mu \mathrm{s})$ of (A) HBP $\left(1.43 \mathrm{mM} ; \varepsilon_{355}=244\right.$ $\left.\mathrm{M}^{-1} \mathrm{~cm}^{-1}\right)$, (B) 4-methoxyBP $\left(7.23 \mathrm{mM} ; \varepsilon_{355}=244\right)$, (C) poly(NIPAM-co-BP) $(5.31 \mathrm{mg}$ containing $2.66 \mu \mathrm{mol}$ BP unit; $\left.\varepsilon_{355}=244\right)$. Saturated amount of poly(NIPAM-co-BP) was used for measurement. 


\section{References}

[S1] Leshem, B.; Sarfati, G.; Novoa, A.; Breslav, I.; Marks, R. S. Luminescence 2004, 19, 69-77.

[S2] Schild, H. G.; Tirrell, D. A. J. Phys. Chem. 1990, 94, 4352-4356.

[S3] Shiraishi, Y.; Saito, N.; Hirai, T. J. Am. Chem. Soc. 2005, 127, 8304-8306.

[S4] Shiraishi, Y.; Saito, N.; Hirai, T. J. Am. Chem. Soc. 2005, 127, 12820-12822.

[S5] Das, K. C.; Das, C. K. Biochem. Biophys. Res. Commun. 2002, 295, 62-66.

[S6] Bontempo, D.; Maynard, H. D. J. Am. Chem. Soc. 2005, 127, 6508-6509.

[S7] Rodgers, M. A. J. J. Am. Chem. Soc. 1983, 105, 6201-6205.

[S8] Nardello, V.; Hervé, M.; Alsters, P. L.; Aubry, J.-M. Adv. Synth. Catal. 2002, 344, 184-191.

[S9] Krieger, I. M.; Mulholland, G. W.; Dickey, C. S. J. Phys. Chem.1967, 71, 1123-1129.

[S10] Wise, D. L.; Houghton, G. Chem. Eng. Sci. 1966, 21, 999-1010.

[S11] Zebger, I.; Poulsen, L.; Gao, Z.; Andersen, L. K.; Ogilby, P. R. Langmuir 2003, 19, $8927-8933$.

[S12] Scurlock, R. D.; Kristiansen, M.; Ogilby, P. R.; Taylor, V. L.; Clough, R. L. Polym. Degradation Stability 1998, 60, 145-159.

[S13] Clough, R. L.; Dillon, M. P.; Iu, K.-K.; Ogilby, P. R. Macromolecules 1989, 22, $3620-3628$.

[S14] Gao, Y.; Baca, A. M.; Wang, B.; Ogilby, P. R. Macromolecules 1994, 27, 7041-7048.

[S15] Monkman, A. P.; Burrows, H. D.; Hamblett, I.; Navaratnam, S.; Scherf, U.; Schmitt, C. Chem. Phys. Lett. 2000, 327, 111-116.

[S16] Schiller, K.; Müller, F. W. Polym. Int. 1991, 25, 19-22.

[S17] Gao, Y.; Ogilby, P. R. Macromolecules 1992, 25, 4962-4966.

[S18] Poulsen, L.; Zebger, I.; Klinger, M.; Eldrup, M.; Sommer-Larsen, P.; Ogilby, P. R. Macromolecules 2003, 36, 7189-7198. 